\title{
Corrigendum
}

\section{Transfer of pathogens to and from patients, healthcare providers and medical devices during care activity - a systematic review and meta-analysis-CORRIGENDUM}

In this issue of the journal, the article by Wolfensberger et $\mathrm{al}^{1}$ was originally incorrectly published as a Review, rather than an Original Article. The article type has since been corrected.

\section{Reference}

1. Wolfensberger A, Clack L, Kuster SP, et al. Transfer of pathogens to and from patients, healthcare providers, and medical devices during care activity-a systematic review and meta-analysis. Infect Control Hosp Epidemiol 2018;39:1093-1107. 